\title{
Coulrophobia: how irrational is fear of clowns?
}

\author{
Lennard T. van Venrooij ${ }^{1}$ Pieter C. Barnhoorn ${ }^{1}$
}

Received: 10 February 2017 / Accepted: 21 March 2017 /Published online: 25 March 2017

(C) Springer-Verlag Berlin Heidelberg 2017

\section{Dear Editor:}

In a large study, Meiri et al. found that $1.2 \%$ of hospitalised children experience a fear of clowns (coulrophobia) [2]. Their findings are very interesting, particularly in light of our recent study, which found that four out of 14 paediatricians and paediatric residents consider themselves afraid of clowns [5]. Meiri and colleagues advocate for more studies on this poorly described phenomenon, and we agree. We would like to share our thoughts on one aspect of the paper by Meiri et al. which may impede the exploration of this phenomenon in future research: the definition of coulrophobia.

Because the term coulrophobia is not listed in the WHO's ICD-10 nor in the APA's DSM-V, Meiri et al. needed to define coulrophobia first [1]. They chose to define coulrophobia as "an irrational fear of clowns". We feel that the use of the word "irrational" in this definition can be called into question. This is important, because consensus on the definition is needed for future studies on this phenomenon (such as research on enhancing hospital clown collaboration with hospital staff in order to improve patient well-being).

In 2016, there were reports of clown sightings worldwide. These so-called killer clowns are menacing-looking clowns who scare unsuspecting passers-by (in e.g. Newcastle upon Tyne, England and Greenville, South Carolina) [3, 4]. This series of events led to a social discussion on public safety, which raised

Revisions received: 12 Feb 2017

Communicated by Jaan Toelen

Lennard T. van Venrooij

1.t.van.venrooij@umail.leidenuniv.nl

1 Department of Public Health and Primary Care, Leiden University Medical Center (LUMC), Leiden, The Netherlands the question of whether the attention paid globally to clowns as frightening figures was overblown or justified. In our opinion, being afraid of something harmless may be irrational, until there is a realistic possibility that which is feared is actually harmful. Defining coulrophobia as always irrational could aggravate the social stigmatisation of people who suffer from this specific phobia. This, in turn, may cause sufferers to face difficulties expressing their anxiety, which could bias study results on coulrophobia prevalences and could also lead to coulrophobic patients not receiving treatment due to their reluctance to seek help.

\section{Compliance with ethical standards}

Disclosures None reported.

\section{References}

1. ICD-10 Now! (2016) F40.298 Other specified phobia. Available at: http://icd10now.com/icd10/code/F40.298. Accessed at 02-02-2017.

2. Meiri N, Schnapp Z, Ankri Itay A, Nahmias I, Raviv A, Sagi O, Hamad Saied M, Konopnicki M, Pillar G (2017) Fear of clowns in hospitalized children: prospective experience. Eur J Pediatr 176(2): 269-272. doi:10.1007/s00431-016-2826-3

3. New York Upstate (2016) 'Creepy clown' epidemic comes to Upstate NY, several sightings reported. Available at: http://www. newyorkupstate.com/capital-region/2016/09/creepy_clown epidemic_comes_to_upstate_ny_several_sightings_reported.html. Accessed at 01-02-2017.

4. The Guardian (2016) Clown sightings: the day the craze began. An epidemic of clown sightings is spreading across America - and no one is laughing any more. Available at: https://www.theguardian. com/culture/2016/oct/05/clown-sightings-south-carolina-alabama. Accessed at 01-02-2017.

5. Van Venrooij LT, Barnhoorn PC (2017) Hospital clowning: a paediatrician's view. Eur J Pediatr 176(2):191-197. doi:10.1007/s00431016-2821-8 